\title{
High-resolution measurements of the vacuum ultraviolet energy levels of trivalent gadolinium by excited state excitation
}

\author{
P. S. Peijzel,* P. Vermeulen, W. J. M. Schrama, and A. Meijerink \\ Debye Institute, Utrecht University, P.O. Box 80 000, 3508 TA Utrecht, The Netherlands \\ M. F. Reid \\ Department of Physics and Astronomy, University of Canterbury, Christchurch, New Zealand \\ G. W. Burdick \\ Department of Physics, Andrews University, Berrien Springs, Michigan 49104, USA
}

(Received 16 October 2004; published 24 March 2005)

\begin{abstract}
The energy levels of lanthanide ions have been studied in great detail in the energy range up to $40000 \mathrm{~cm}^{-1}$ $(250 \mathrm{~nm})$. Recently an increased interest in the high energy levels between 40000 and $70000 \mathrm{~cm}^{-1}$ has emerged, partly triggered by the need for new luminescent materials for vacuum ultraviolet (VUV) excitation. Using synchrotron radiation many new energy levels have been discovered for various lanthanide ions. However, the spectral resolution of a synchrotron is limited and to resolve the complete energy level structure higher resolution tunable lasers are required. Unfortunately no high-resolution tunable lasers are available in the VUV. To overcome this problem two-photon spectroscopy may be applied. In this paper resonant twophoton spectroscopy is applied to measure the energy level structure of $\mathrm{Gd}^{3+}$ in fluorides. Excited state excitation (ESE) from the ${ }^{6} P_{7 / 2}$ level is shown to provide high-resolution spectra of the high energy levels of $\mathrm{Gd}^{3+}$. The extension of the energy level structure is used to improve energy level calculations, which is especially beneficial for $\mathrm{Gd}^{3+}$ where only a limited number of energy levels is available from conventional laser spectroscopy.
\end{abstract}

DOI: $10.1103 /$ PhysRevB.71.125126

PACS number(s): 78.40.Ha, 78.20.Bh, 71.55.-i, 71.70.Ch

\section{INTRODUCTION}

In the past decades the spectroscopic properties of the trivalent gadolinium ion has been studied extensively using one-photon and two-photon excitation spectroscopy. As a result, the positions of the energy levels up to $40000 \mathrm{~cm}^{-1}$ in several host-lattices are known very accurately. ${ }^{1-3,5-7}$ The parameters required for energy level calculations are obtained by fitting the calculated levels to experimental values. ${ }^{1,2}$ This works well for the lower energy levels, but to accurately calculate the higher energy levels of gadolinium situated in the vacuum ultraviolet (VUV, >50 $000 \mathrm{~cm}^{-1}$ ) experimental values from that spectral region are required.

Recently many of the VUV levels of gadolinium have been measured using synchrotron radiation ${ }^{8}$ but the spectral resolution of these experiments is not high enough to resolve the splitting of several multiplets into all crystal field components and therefore an unambiguous assignment of measured energy levels to the calculated levels is not possible.

To obtain higher resolution spectra in the UV and VUV a laser is required, but unfortunately no high-resolution tunable VUV lasers exist at the moment. To overcome this problem, excited state excitation or two-photon excitation measurements can be used to probe the VUV levels with the high resolution required to resolve individual crystal field levels. In the case of excited state excitation, two tunable lasers are used simultaneously and the gadolinium ion is excited in two steps. The first excitation is at a fixed energy exciting the $\mathrm{Gd}^{3+}$ ion into the ${ }^{6} P_{7 / 2}$ level at about $32200 \mathrm{~cm}^{-1}$, while the second tunable laser is used to excite the $\mathrm{Gd}^{3+}$ ion from the ${ }^{6} P_{7 / 2}$ state to a higher energy state. Commercially available laser dyes allow excitation to levels up to approximately $62000 \mathrm{~cm}^{-1}$ for the trivalent gadolinium ion.

For a high resolution measurement in the $(\mathrm{V}) \mathrm{UV}$ region of the spectrum nonlinear two-photon excitation can be used, where two lower energy photons from one tunable laser are absorbed to excite into the (V)UV levels of gadolinium at twice the photon energy. Due to the inversion of the parity selection rule (intraconfigurational $4 f^{n} \rightarrow 4 f^{n}$ transitions become parity-allowed and interconfigurational $4 f^{n} \rightarrow 4 f^{n-1} 5 d$ transitions become parity-forbidden) this technique is very useful to study $4 f^{n}$ energy levels that are obscured by the strong parity allowed $4 f^{n} \rightarrow 4 f^{n-1} 5 d$ absorption band and has been used to study the $4 f^{n}$ levels of $\mathrm{Eu}^{2+}$ in detail., 3 The disadvantage of this technique is that it is very sensitive to impurities in the samples due to the very low transition probabilities for the nonlinear two-photon excitation process. The probability for resonant two-photon absorption involving an impurity ion is several orders of magnitude higher than the probability of two-photon excitation. The method of excited state excitation does not suffer from this problem since the first excitation step is very specific for the ion to be probed. In this contribution we present our results using the technique of excited state excitation to measure high energy levels of $\mathrm{Gd}^{3+}$ in $\mathrm{LaF}_{3}$. It is shown that excited state excitation can be applied to measure the energies of the $4 f^{n}$ levels in the VUV with high accuracy $\left(\sim 1 \mathrm{~cm}^{-1}\right)$. Better knowledge of these levels is used to improve calculation parameters. 


\section{EXCITED STATE EXCITATION}

In order to measure and resolve all crystal field components within the $4 f^{n}$ configuration of lanthanide ions an excitation source with a high spectral resolution (typically in the order of $1 \mathrm{~cm}^{-1}$, the inhomogeneous linewidth) is required. Since these transitions are parity-forbidden, also a high intensity is needed. Tunable dye lasers meet these criteria and have been used since the 1970s to resolve the energy level structure of lanthanide ions in the near-infrared, visible and ultraviolet in a wide variety of crystals. Unfortunately there are no high-resolution tunable lasers in the VUV yet, so one photon absorption is not an option to measure the high $4 f^{n}$ energy levels of lanthanide ions. Synchrotrons do provide a high intensity and tunable VUV excitation source, but the resolution of a typical $1 \mathrm{~m}$ monochromator is about $0.3 \AA\left(\sim 10 \mathrm{~cm}^{-1}\right.$ in the spectral region between 150 and $200 \mathrm{~nm}$ ) which is not enough to resolve all crystal field levels. Nevertheless, the highest resolution excitation spectra in the VUV have been recorded using synchrotron radiation. ${ }^{8-11}$ The use of excited state excitation, using tunable dye lasers with a resolution better than $1 \mathrm{~cm}^{-1}$, offers the possibility to study the VUV levels of lanthanide ions with sufficient resolution to resolve all crystal field components.

In contrast with direct two-photon absorption, excited state excitation involves no virtual intermediate state. Instead, the ion under investigation is excited to a metastable intermediate energy level, followed by a second excitation to higher energy levels. Next, multiphonon relaxation occurs until an emitting level, having an energy gap to the next lower level of more than 4 to 5 times the maximum phonon energy of that lattice, is reached and emission from this level is observed. When the emitting level is situated above the first metastable level, anti-Stokes emission is observed when excited state excitation takes place.

The trivalent gadolinium ion has a suitable energy level scheme for excited state excitation measurements (see Fig. 1). First, excitation around $310 \mathrm{~nm}$ brings the ion to the ${ }^{6} P_{7 / 2}$ state. After absorption of a second photon and (possibly) multiphonon relaxation, emission occurs from the ${ }^{6} I_{7 / 2}$, ${ }^{6} D_{9 / 2}$, and ${ }^{6} G_{7 / 2}$ levels at, respectively, $272 \mathrm{~nm}, 252 \mathrm{~nm}$, and $203 \mathrm{~nm}$. Detection of one of these three anti-Stokes emissions can then be used as a probe for excited state excitation.

Excited state excitation measurements have some advantages compared to two-photon excitation. First, the transition probabilities are higher as the oscillator strength is about 6 orders of magnitude higher. Second, impurities are less of a problem when the ion under investigation is being excited selectively. Tuning of the first excitation laser to an energy that corresponds to a resonant transition of the $\mathrm{Gd}^{3+}$ ion (in Fig. 1(a) ${ }^{8} S_{7 / 2} \rightarrow{ }^{6} P_{7 / 2}$ transition) gives the two-photon excitation process for the $\mathrm{Gd}^{3+}$ ion a strong enhancement over undesired two-photon excitation processes involving impurities or defects.

In the case of gadolinium another advantage is that the first excited state $\left({ }^{6} P_{7 / 2}\right)$ is a spin sextet. Transitions to the higher sextets and quartets have a higher transition probability than transitions to these states starting from the octet ${ }^{8} S_{7 / 2}$ ground state of $\mathrm{Gd}^{3+}$.

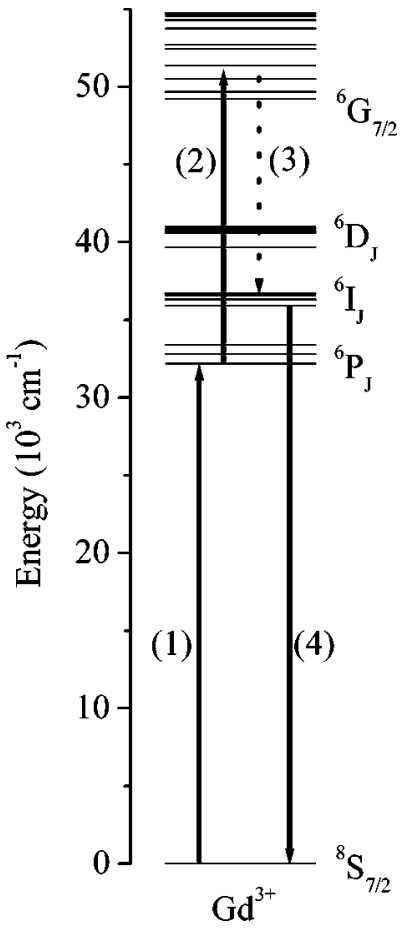

FIG. 1. Energy level scheme showing free-ion levels for $\mathrm{Gd}^{3+}$ up to $55000 \mathrm{~cm}^{-1}$ with arrows indicating the transitions for excited state excitation. (1) Excitation into the ${ }^{6} P_{7 / 2}$ level, (2) excitation using a tunable laser, (3) relaxation to the ${ }^{6} I_{7 / 2}$ level, and (4) antiStokes emission from the ${ }^{6} I_{7 / 2}$ level.

\section{EXPERIMENT}

\section{A. Sample preparation}

A single crystal of $\mathrm{LaF}_{3}$ doped with $0.5 \% \mathrm{Gd}^{3+}$ was grown in a Philips PH 1006/13 high-frequency furnace using the vertical Bridgman method. A slice of approximately $7 \mathrm{~mm}$ in diameter and with a thickness of $2 \mathrm{~mm}$ was cut and polished and glued to a copper sample holder.

\section{B. Excited state excitation}

Excited state excitation measurements were performed at approximately $10 \mathrm{~K}$ using an Air Products APC HC-2 cold finger type closed cycle cryostat fitted with quartz windows. The experimental setup for ESE consisted of two tunable lasers. The first was a Spectra-Physics PDL3 dye laser pumped by a frequency doubled Spectra-Physics Quanta Ray $\mathrm{Nd}$ :YAG laser operating at $15 \mathrm{~Hz}$. A Rhodamine B laser dye solution was used to obtain a laser tunable around $620 \mathrm{~nm}$. This red laser beam was frequency doubled to a UV laser to a wavelength around $310 \mathrm{~nm}$ by a Spectra-Physics wavelength extender with KDP crystals. For all ESE spectra reported in this paper, the wavelength of this laser was fixed at $310.25 \mathrm{~nm}$, corresponding to the transition of the ${ }^{8} S_{7 / 2}$ ground state to the highest crystal field component of the ${ }^{6} P_{7 / 2}$ level. The second laser used was a Lambda Physik LPD 3002 Dye laser which was pumped with a Lambda Physik LPX $100 \mathrm{XeCl}$ excimer laser. This laser was used to scan a wavelength range determined by the dyes used. In Table I an overview of the various dyes is given together with the en- 
TABLE I. Laser dyes used for excited state excitation measurements. The excitation region that is reached by the sum of the energies of the UV laser $(310 \mathrm{~nm})$ and the second dye laser is indicated in the last column.

\begin{tabular}{ccc}
\hline \hline Dye & $\begin{array}{c}\text { Wavelength } \\
(\mathrm{nm})\end{array}$ & $\begin{array}{c}\text { Excitation } \\
\text { region }\left(\mathrm{cm}^{-1}\right)\end{array}$ \\
\hline Rhodamine 6G & $570-600$ & $48900-49700$ \\
Coumarine 153 & $520-580$ & $49400-51400$ \\
Coumarine 307 & $470-540$ & $50700-53500$ \\
Coumarine 120 & $430-470$ & $53500-55500$ \\
Bis-MSB & $403-436$ & $55100-57000$ \\
PBBO & $388-420$ & $56000-58000$ \\
BiBuQ & $375-390$ & $57800-58800$ \\
DMQ & $350-385$ & $58100-60700$ \\
PTP & $340-355$ & $60400-61600$ \\
\hline \hline
\end{tabular}

ergy range that can be probed by ESE from the ${ }^{6} P_{7 / 2}$ level. A Stanford Research Systems DG535 digital delay/pulse generator was used to trigger the YAG laser and excimer laser with a delay of $10 \mu$ s. Both laser beams were focussed on the same spot in the crystal using mirrors and lenses. The emission from the crystal was collected using a Hamamatsu R7154 solar blind photomultiplier tube, which was cooled to $-30{ }^{\circ} \mathrm{C}$. Various band and interference filters were used to block any light within the wavelength range of the excitation sources. The spectra obtained were corrected for the intensity of the dye laser using dye output spectra recorded with a power meter.

\section{RESULTS AND DISCUSSION}

This section describes the excited state excitation spectra and the assignment of the experimentally observed energy levels to an energy level scheme calculated for $\mathrm{Gd}^{3+}$ in $\mathrm{LaF}_{3}$. For a recent detailed description of the Hamiltonian and the calculation program that was used, see Ref. 12. The calculated energy level scheme is based on the parameter values listed by Carnall et al. ${ }^{2}$ based on 70 experimentally observed energy levels in the energy region up to $50000 \mathrm{~cm}^{-1}$. In Carnall's energy level fittings, the $B_{q}^{k}$ crystal field parameter values were not varied, but were fixed at the parameter values for terbium. In order to make a comparison of parameter values based on fitting of the lower energy (UV) levels with the parameter values obtained from fittings including the VUV energy levels possible, we refitted the data of Carnall et al. We performed a least squares fitting of the $B_{q}^{k}$ for all 70 experimentally observed levels up to $50000 \mathrm{~cm}^{-1}$ as listed by Carnall et al. Only the cylindrically symmetric $B_{0}^{k}$,s were allowed to vary, the other crystal field parameters were kept at fixed (terbium) ratios to the $B_{0}^{k}$ values. Using 70 data points and varying 10 parameters a fit with a standard deviation of $8.0 \mathrm{~cm}^{-1}$ was obtained. As expected, the quality of the fit has improved (smaller $\sigma$ and rms) by allowing the variation of the $B_{0}^{k}$ parameter values. All parameter values are listed together with their uncertainties in Table II.
TABLE II. Free-ion and crystal field parameters for $\mathrm{LaF}_{3}: \mathrm{Gd}^{3+}$ based on fitting the lower energy (UV) levels of $\mathrm{Gd}^{3+}$. The left column shows the parameters reported by Carnall et al. (Ref. 2) and the right column shows the parameters obtained by fitting to the experimental energy levels reported by Carnall for $\mathrm{LaF}_{3}: \mathrm{Gd}^{3+}$ as described in the text. Parameters for which no uncertainty is given were not varied.

\begin{tabular}{|c|c|c|}
\hline Parameter & $\begin{array}{c}\text { Carnall } \\
\text { value }\left(\mathrm{cm}^{-1}\right)\end{array}$ & $\begin{array}{c}\text { New fit } \\
\text { value }\left(\mathrm{cm}^{-1}\right)\end{array}$ \\
\hline$E_{\mathrm{AVG}}$ & & $87812(11)$ \\
\hline$F^{2}$ & $85669(17)$ & $85592(18)$ \\
\hline$F^{4}$ & 60825 & $61015(29)$ \\
\hline$F^{6}$ & $44776(24)$ & $44745(25)$ \\
\hline$\alpha$ & $18.92(0.83)$ & $19.14(0.10)$ \\
\hline$\beta$ & -600 & -600 \\
\hline$\gamma$ & 1575 & 1575 \\
\hline$T^{2}$ & 300 & 300 \\
\hline$T^{3}$ & 42 & 42 \\
\hline$T^{4}$ & 62 & 62 \\
\hline$T^{6}$ & -295 & -295 \\
\hline$T^{7}$ & 350 & 350 \\
\hline$T^{8}$ & 310 & 310 \\
\hline$\zeta$ & $1508(2)$ & $1499(2)$ \\
\hline$M^{0}$ & $3.22(0.2)$ & $3.20(0.03)$ \\
\hline$M^{2}$ & $0.56 M^{0}$ & $0.56 M^{0}$ \\
\hline$M^{4}$ & $0.31 M^{0}$ & $0.31 M^{0}$ \\
\hline$P^{2}$ & $676(75)$ & 676 \\
\hline$P^{4}$ & $0.5 P^{2}$ & $0.5 P^{2}$ \\
\hline$P^{6}$ & $0.1 P^{2}$ & $0.1 P^{2}$ \\
\hline$B_{0}^{2}$ & -231 & $-212(14)$ \\
\hline$B_{0}^{4}$ & 604 & $579(40)$ \\
\hline$B_{0}^{6}$ & 280 & $292(22)$ \\
\hline$B_{2}^{2}$ & -99 & $0.4286 B_{0}^{2}$ \\
\hline$B_{2}^{4}$ & 340 & $0.5629 B_{0}^{4}$ \\
\hline$B_{4}^{4}$ & 452 & $0.7483 B_{0}^{4}$ \\
\hline$B_{2}^{6}$ & -721 & $-2.575 B_{0}^{6}$ \\
\hline$B_{4}^{6}$ & -204 & $-0.7286 B_{0}^{6}$ \\
\hline$B_{6}^{6}$ & -509 & $-1.8179 B_{0}^{6}$ \\
\hline$N$ & 70 & 70 \\
\hline$\sigma$ & 10 & 8 \\
\hline $\mathrm{rms}$ & 9 & 7 \\
\hline
\end{tabular}

\section{A. Excited state excitation spectra}

A typical example of an excited state excitation spectrum is shown in Fig. 2. This spectrum shows absorptions to the four crystal field levels of the ${ }^{4} D(6)_{7 / 2}$ state and the absorption to the ${ }^{6} F_{1 / 2}$ level, which cannot be split by the crystal field because $J=1 / 2$. In the spectrum in Fig. 2 more than the five expected transitions are observed. Upon excitation into the ${ }^{6} P_{7 / 2}$ multiplet, fast relaxation to the lowest crystal field level occurs. The other three crystal field levels are thermally populated according to a Boltzmann distribution. Especially 


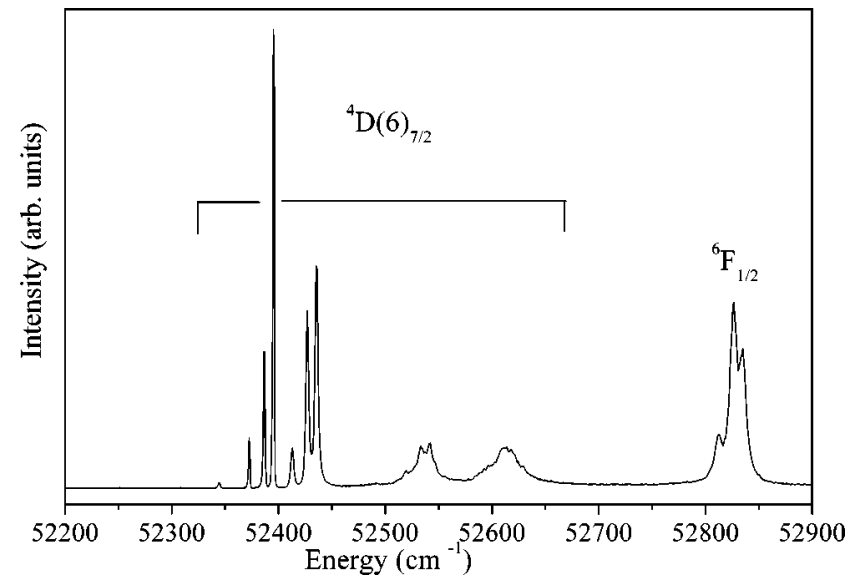

FIG. 2. Excited state excitation spectrum of the ${ }^{4} D(6)_{7 / 2}$ and ${ }^{6} \mathrm{~F}_{1 / 2}$ levels of $\mathrm{LaF}_{3}: \mathrm{Gd}^{3+}$ monitoring ${ }^{6} I_{7 / 2}$ emission at $10 \mathrm{~K}$.

the second level, which is situated only $8 \mathrm{~cm}^{-1}$ above the lowest level (in $\mathrm{LaF}_{3}$ ), is still considerably populated (24\%) at a temperature of $10 \mathrm{~K}$.

The population of the higher crystal field levels gives rise to more absorption lines in the excited state excitation spectrum, since excitations from the thermally populated levels can also occur. This results in extra absorption lines at energy separations equal to the splitting of the ${ }^{6} P_{7 / 2}$ levels. In some cases it is easy to recognize the difference between these transitions since the energy separations between the ${ }^{6} P_{7 / 2}$ levels are known exactly. If the energy levels are closer together than about $50 \mathrm{~cm}^{-1}$ which is the total splitting of the ${ }^{6} P_{7 / 2}$ levels, absorption peaks will overlap and the spectra should be interpreted more carefully. Although the populations of the four ${ }^{6} P_{7 / 2}$ levels can be estimated using a Boltzmann-distribution, it is not possible to relate this to the relative absorption intensities since they also depend on symmetry and polarization. This is clearly visible for the absorption to the ${ }^{6} F_{1 / 2}$ level at about $52800 \mathrm{~cm}^{-1}$, where the transition originating from the second ${ }^{6} P_{7 / 2}$ crystal field level is the most intense transition. In Table III the experimental energies of the ${ }^{4} D(6)_{7 / 2}$ and ${ }^{6} F_{1 / 2}$ levels are listed together with the calculated energies and $M_{J}$ values for these levels using parameters from Table II. As the calculated energy levels contain contributions of wave functions of several states, the $M_{J}$ value having the largest contribution is listed. The experimental energies of the levels were determined by adding the

TABLE III. Experimentally observed and calculated energies of the ${ }^{4} D(6)_{7 / 2}$ and ${ }^{6} F_{1 / 2}$ levels of $\mathrm{Gd}^{3+}$ in $\mathrm{LaF}_{3}$. The calculations are based on the new fit parameters from Table II.

\begin{tabular}{ccccc}
\hline \hline Energy level & $M_{J}$ & $\begin{array}{c}E_{\mathrm{EXP}} \\
\left(\mathrm{cm}^{-1}\right)\end{array}$ & $\begin{array}{c}E_{\mathrm{CALC}} \\
\left(\mathrm{cm}^{-1}\right)\end{array}$ & $\begin{array}{c}E_{\mathrm{EXP}}-E_{\mathrm{CALC}} \\
\left(\mathrm{cm}^{-1}\right)\end{array}$ \\
\hline${ }^{4} D(6)_{7 / 2}$ & $\pm 5 / 2$ & 52396 & 52356 & 40 \\
${ }^{4} D(6)_{7 / 2}$ & $\pm 3 / 2$ & 52436 & 52370 & 66 \\
${ }^{4} D(6)_{7 / 2}$ & $\pm 1 / 2$ & 52541 & 52490 & 51 \\
${ }^{4} D(6)_{7 / 2}$ & $\pm 7 / 2$ & 52617 & 52554 & 63 \\
${ }^{6} F_{1 / 2}$ & $\pm 1 / 2$ & 52835 & 52729 & 106 \\
\hline \hline
\end{tabular}

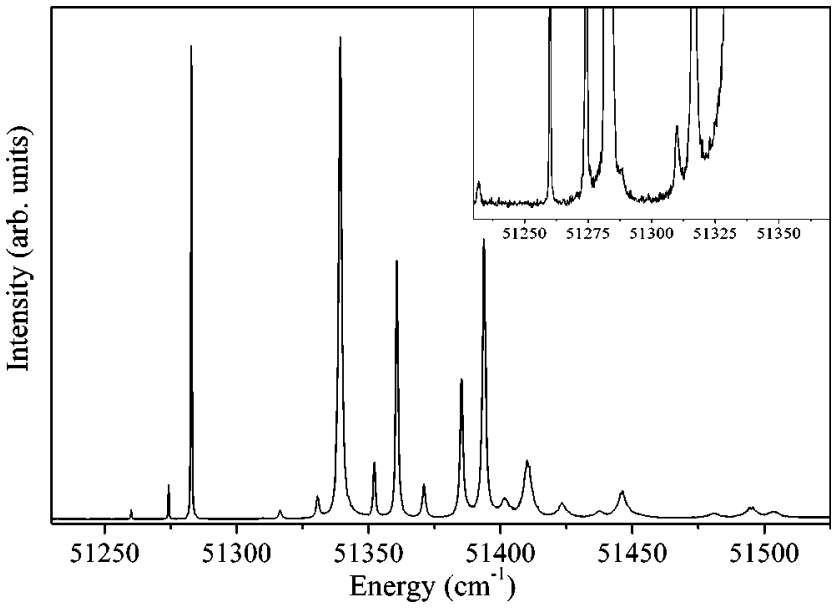

FIG. 3. Excited state excitation spectrum of the ${ }^{6} G_{13 / 2}$ levels of $\mathrm{LaF}_{3}: \mathrm{Gd}^{3+}$ monitoring ${ }^{6} I_{7 / 2}$ emission at $10 \mathrm{~K}$. The inset shows a magnified view of the region $51230-51350 \mathrm{~cm}^{-1}$.

energy of the lowest ${ }^{6} P_{7 / 2}$ level to the energies of the peaks in the excited state excitation spectrum that corresponds to excited state excitation from the lowest ${ }^{6} P_{7 / 2}$ level $\left(32182 \mathrm{~cm}^{-1}\right)$. In all excited state excitation spectra shown in this paper, this energy has been added to the values on the $x$-axis.

All experimentally observed energies are around 40 to $100 \mathrm{~cm}^{-1}$ higher compared to the calculated energies. This clearly shows that there is a mismatch between experiment and calculation for the higher energy levels when only a set of low energy levels is used to fit the parameters. Although there is a significant difference between the calculated and observed energies, assignment of these levels is still possible since there is an energy gap of $1000 \mathrm{~cm}^{-1}$ above and below these levels.

A second effect, besides the observation of more lines due to thermal population of the ${ }^{6} P_{7 / 2}$ crystal field components, that complicates the determination of all VUV energy levels from the excited state excitation spectra is broadening of some of the absorption peaks. This is clearly visible in the spectrum shown in Fig. 3 which shows all seven crystal field levels of the ${ }^{6} G_{13 / 2}$ multiplet. The broadening in the spectrum is probably caused by lifetime broadening due to fast relaxation, which is expected when there are energy levels below the one investigated at energy separations of approximately $100-350 \mathrm{~cm}^{-1}$. The relaxation rate, at low temperatures, is due to a phonon emission process. The highest relaxation rates are for one-phonon emission. The rate is dependent on the density of states in the phonon spectrum. The phonon density of states increases with energy (in the Debye model the density of states is proportional to $\omega^{2}$ ) which explains the lower limit of $100 \mathrm{~cm}^{-1}$. The phonon cutoff energy in $\mathrm{LaF}_{3}$ is about $350 \mathrm{~cm}^{-1}$. For bridging energy gaps higher than $350 \mathrm{~cm}^{-1}$ multiphonon emission processes are involved which have lower rates. A more quantitative analysis of the linewidth is beyond the scope of the present work. The broadening effect is illustrated in Fig. 3, where the lowest crystal field level of the ${ }^{6} G_{13 / 2}$ multiplet is observed at $51280 \mathrm{~cm}^{-1}$. Broadening of the absorption peaks is expected starting at about $51400 \mathrm{~cm}^{-1}$, which is observed in the spec- 
TABLE IV. Experimentally observed and calculated energies of the seven ${ }^{6} G_{13 / 2}$ levels of $\mathrm{Gd}^{3+}$ in $\mathrm{LaF}_{3}$. For the calculation the new fit parameters from Table II were used. The crystal field levels of the ${ }^{6} P_{7 / 2}$ multiplet from which the absorption originates is also indicated, where number 1 indicates the lowest level.

\begin{tabular}{cccc}
\hline \hline${ }^{6} G_{13 / 2}$ level & $\begin{array}{c}E_{\mathrm{EXP}} \\
\left(\mathrm{cm}^{-1}\right)\end{array}$ & $\begin{array}{c}E_{\mathrm{CALC}} \\
\left(\mathrm{cm}^{-1}\right)\end{array}$ & $\begin{array}{c}{ }^{6} P_{7 / 2} \\
\text { origin }\end{array}$ \\
\hline 1 & 51232 & & 4 \\
1 & 51260 & & 3 \\
1 & 51274 & & 2 \\
1 & 51283 & 51269 & 1 \\
2 & 51288 & & 4 \\
3 & 51310 & & 4 \\
2 & 51316 & & 3 \\
2 & 51331 & & 2 \\
2 & 51339 & 51319 & 1 \\
3 & 51352 & & 1 \\
3 & 51361 & 51346 & 3 \\
4 & 51371 & & 2 \\
4 & 51385 & & 1 \\
4 & 51393 & 51366 & 2 \\
5 & 51404 & & 1 \\
5 & 51410 & 51385 & 3 \\
6 & 51423 & & 2 \\
6 & 51438 & & 1 \\
6 & 51447 & 51400 & \\
7 & 51481 & & \\
7 & 51496 & & \\
7 & 51503 & 51450 & \\
\hline
\end{tabular}

trum. In Table IV the experimentally determined energies are listed together with the calculated energies for the ${ }^{6} G_{13 / 2}$ levels using parameters from Table II. The line broadening causes a weaker maximum absorption and overlapping of absorption peaks which hampers the observation and assignment of many transitions, especially in the energy region above $55000 \mathrm{~cm}^{-1}$. The assignments in Table IV are based on the observation of the splitting pattern of the ${ }^{6} P_{7 / 2}$ level from which excitation occurs. The difference between calculated and experimental energies increases going from the lowest to the highest multiplet level.

Another phenomenon that influences absorption intensities is the spin selection rule. The excited state from which excited state excitation occurs $\left({ }^{6} P_{7 / 2}\right)$ is a spin sextet. Transitions to other sextets are spin-allowed and are more intense than transitions to spin-quartet levels, and transitions to spindoublet levels are expected to be too weak to be observed. At energies of $55900 \mathrm{~cm}^{-1}$ and higher spin quartet levels are calculated, above $67000 \mathrm{~cm}^{-1}$ spin doublet states are also calculated. For lanthanide ions the spin selection rule is lifted by the strong spin-orbit coupling which mixes states of the same $J$ value but with different spin multiplicity. As a result, the influence of the spin selection rule is a general trend while for a better understanding of the intensities it is necessary to consider all states contributing to a certain level that

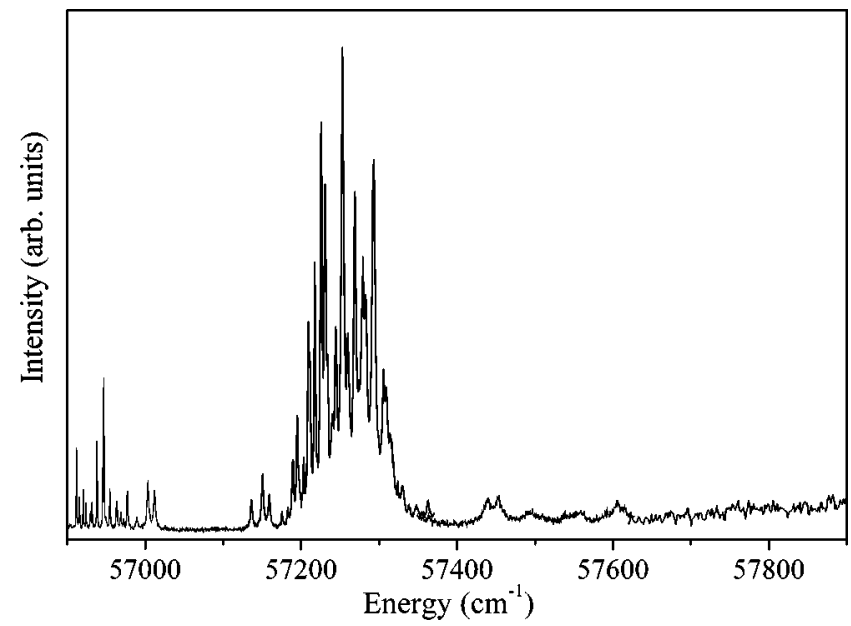

FIG. 4. Excited state excitation spectrum of $\mathrm{LaF}_{3}: \mathrm{Gd}^{3+}$ in the region $56900-57900 \mathrm{~cm}^{-1}$ monitoring ${ }^{6} I_{7 / 2}$ emission at $10 \mathrm{~K}$. Two different laser dyes were used for recording this spectrum with an overlap around $57300 \mathrm{~cm}^{-1}$.

is labeled by the term symbol for the state with the largest contribution.

Figure 4 shows the ESE spectrum for the region $56900-57900 \mathrm{~cm}^{-1}$. In the region $57450-57820 \mathrm{~cm}^{-1} 16$ levels are calculated for which the two largest contributions are from spin quartet states. Only some weak absorptions are observed, and it is not possible to assign these to specific calculated energy levels. The low intensities of the absorption peaks in this region is related to the quartet spin character of the final states. Also for the region $56900-57000 \mathrm{~cm}^{-1}$ levels with a considerable sextet character are calculated and observed.

In lanthanide spectroscopy $J$ is a better quantum number and $J$-selection rules apply. For electric dipole transitions $\Delta J$ is limited to a maximum of \pm 6 . This means that electric dipole transitions originating from the ${ }^{6} P_{7 / 2}$ state are allowed up to levels with a $J$ of $19 / 2$. The levels with the highest $J$ levels for $\mathrm{Gd}^{3+}$ are the ${ }^{2} Q_{23 / 2}$ and ${ }^{2} Q_{25 / 2}$ level. These levels cannot be observed, due to a $\Delta J$ of 8 or 9 and a change in spin of 2.

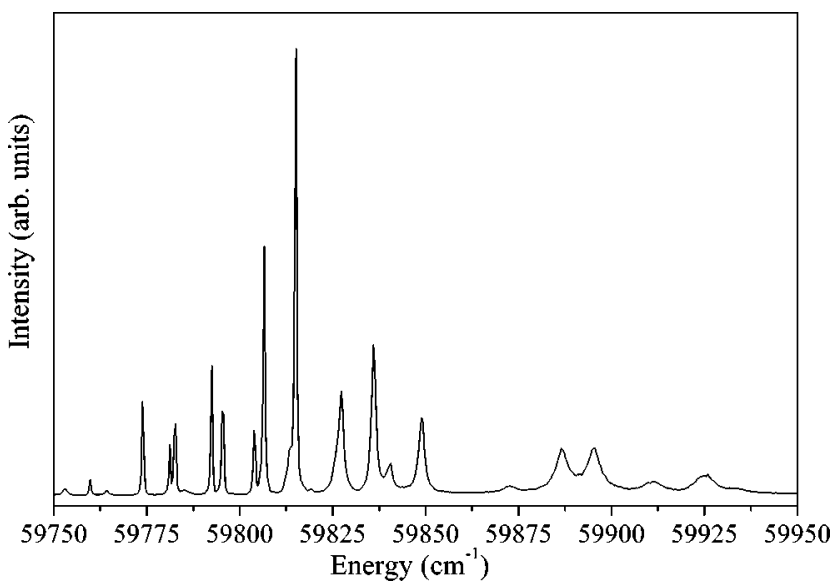

FIG. 5. Excited state excitation spectrum of the ${ }^{6} H_{13 / 2}$ multiplet in $\mathrm{LaF}_{3}: \mathrm{Gd}^{3+}$ in the region $59750-59950 \mathrm{~cm}^{-1}$ monitoring ${ }^{6} I_{7 / 2}$ emission at $10 \mathrm{~K}$. 
TABLE V. Experimentally observed and calculated energies of the ${ }^{6} H_{13 / 2}$ levels of $\mathrm{Gd}^{3+}$ in $\mathrm{LaF}_{3}$. The calculations are based on the new fit parameters from Table II.

\begin{tabular}{cccc}
\hline \hline$M_{J}$ & $\begin{array}{c}E_{\mathrm{EXP}} \\
\left(\mathrm{cm}^{-1}\right)\end{array}$ & $\begin{array}{c}E_{\mathrm{CALC}} \\
\left(\mathrm{cm}^{-1}\right)\end{array}$ & $\begin{array}{c}E_{\mathrm{EXP}}-E_{\mathrm{CALC}} \\
\left(\mathrm{cm}^{-1}\right)\end{array}$ \\
\hline $\pm 13 / 2$ & 59781 & 59658 & 123 \\
$\pm 7 / 2$ & 59804 & 59679 & 125 \\
$\pm 5 / 2$ & 59815 & 59699 & 116 \\
$\pm 1 / 2$ & 59836 & 59708 & 128 \\
$\pm 11 / 2$ & 59849 & 59721 & 128 \\
$\pm 9 / 2$ & 59895 & 59763 & 132 \\
$\pm 3 / 2$ & 59933 & 59798 & 135 \\
\hline \hline
\end{tabular}

Since not all energy levels could be assigned, especially for the levels above $54000 \mathrm{~cm}^{-1}$ it was not possible to include all levels in the fitting of the energy levels in order to obtain new parameter values. Energy levels could only be assigned unambiguously if all crystal field levels of an isolated multiplet were observed. As an example, Fig. 5 shows the absorptions of the ${ }^{6} H_{13 / 2}$ multiplet, for which all seven crystal field levels are observed. The calculated and experimentally obtained energies of the ${ }^{6} H_{13 / 2}$ levels are listed in Table V. The difference between experimental and calculated energies varies between 115 and $135 \mathrm{~cm}^{-1}$. This indicates a shift of the whole multiplet, while the crystal field splitting is good.

The highest two multiplets observed are the ${ }^{4} F(4)_{9 / 2}$ and ${ }^{4} F(4)_{7 / 2}$ levels in the region 60900 to $61400 \mathrm{~cm}^{-1}$. The spectrum in Fig. 6 shows all crystal field levels and their energies, which are collected in Table VI.

In the next section the fitting procedure will be discussed leading to improved parameter values by including the presently observed high energy (VUV) levels of $\mathrm{Gd}^{3+}$ in $\mathrm{LaF}_{3}$.

The lower spectrum in Fig. 7 shows the excited state excitation spectrum for the complete range of dyes used. The energy on the horizontal axis is the energy at which excited state excitation peaks are observed added to $32182 \mathrm{~cm}^{-1}$, the

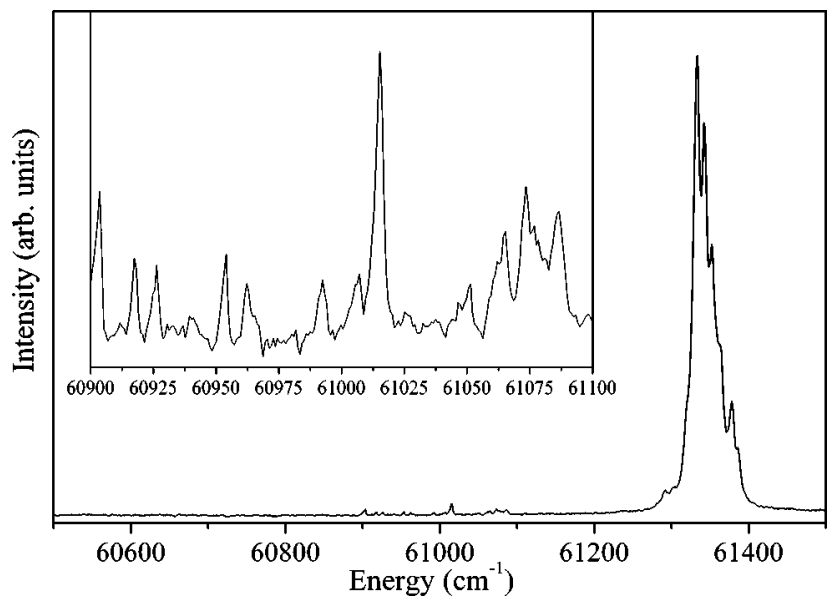

FIG. 6. Excited state excitation spectrum of the ${ }^{4} F(4)_{9 / 2}$ and ${ }^{4} F(4)_{7 / 2}$ multiplets in $\mathrm{LaF}_{3}: \mathrm{Gd}^{3+}$ in the region $60900-61400 \mathrm{~cm}^{-1}$ monitoring ${ }^{6} I_{7 / 2}$ emission at $10 \mathrm{~K}$.
TABLE VI. Experimentally observed and calculated energies of the ${ }^{4} F(4)_{9 / 2}$ and ${ }^{4} F(4)_{7 / 2}$ levels of $\mathrm{Gd}^{3+}$ in $\mathrm{LaF}_{3}$. The calculations are based on the new fit parameters from Table II.

\begin{tabular}{ccccc}
\hline \hline Energy level & $M_{J}$ & $\begin{array}{c}E_{\mathrm{EXP}} \\
\left(\mathrm{cm}^{-1}\right)\end{array}$ & $\begin{array}{c}E_{\mathrm{CALC}} \\
\left(\mathrm{cm}^{-1}\right)\end{array}$ & $\begin{array}{c}E_{\mathrm{EXP}}-E_{\mathrm{CALC}} \\
\left(\mathrm{cm}^{-1}\right)\end{array}$ \\
\hline${ }^{4} F(4)_{9 / 2}$ & $\pm 7 / 2$ & 60927 & 60773 & 154 \\
${ }^{4} F(4)_{9 / 2}$ & $\pm 5 / 2$ & 60964 & 60839 & 125 \\
${ }^{4} F(4)_{9 / 2}$ & $\pm 1 / 2$ & 61016 & 60860 & 156 \\
${ }^{4} F(4)_{9 / 2}$ & $\pm 3 / 2$ & 61075 & 60921 & 154 \\
${ }^{4} F(4)_{9 / 2}$ & $\pm 9 / 2$ & 61088 & 60944 & 144 \\
${ }^{4} F(4)_{7 / 2}$ & $\pm 5 / 2$ & 61334 & 61208 & 126 \\
${ }^{4} F(4)_{7 / 2}$ & $\pm 7 / 2$ & 61344 & 61213 & 131 \\
${ }^{4} F(4)_{7 / 2}$ & $\pm 1 / 2$ & 61365 & 61226 & 139 \\
${ }^{4} F(4)_{7 / 2}$ & $\pm 3 / 2$ & 61388 & 61245 & 143 \\
\hline \hline
\end{tabular}

energy of the lowest ${ }^{6} P_{7 / 2}$ level. The spectrum has been corrected for the laser intensity using correction files recorded for each dye. The spectra for correction for the intensity of the UV dyes could not be measured and the intensity of the ESE spectrum in the region above $57000 \mathrm{~cm}^{-1}$ has been scaled to the efficiencies of the dyes used. This results in an estimated error in intensity of $10 \%$ to $20 \%$. Furthermore, some wavelength regions were obtained using the same dye, but with a different grating order of the laser. This leads to

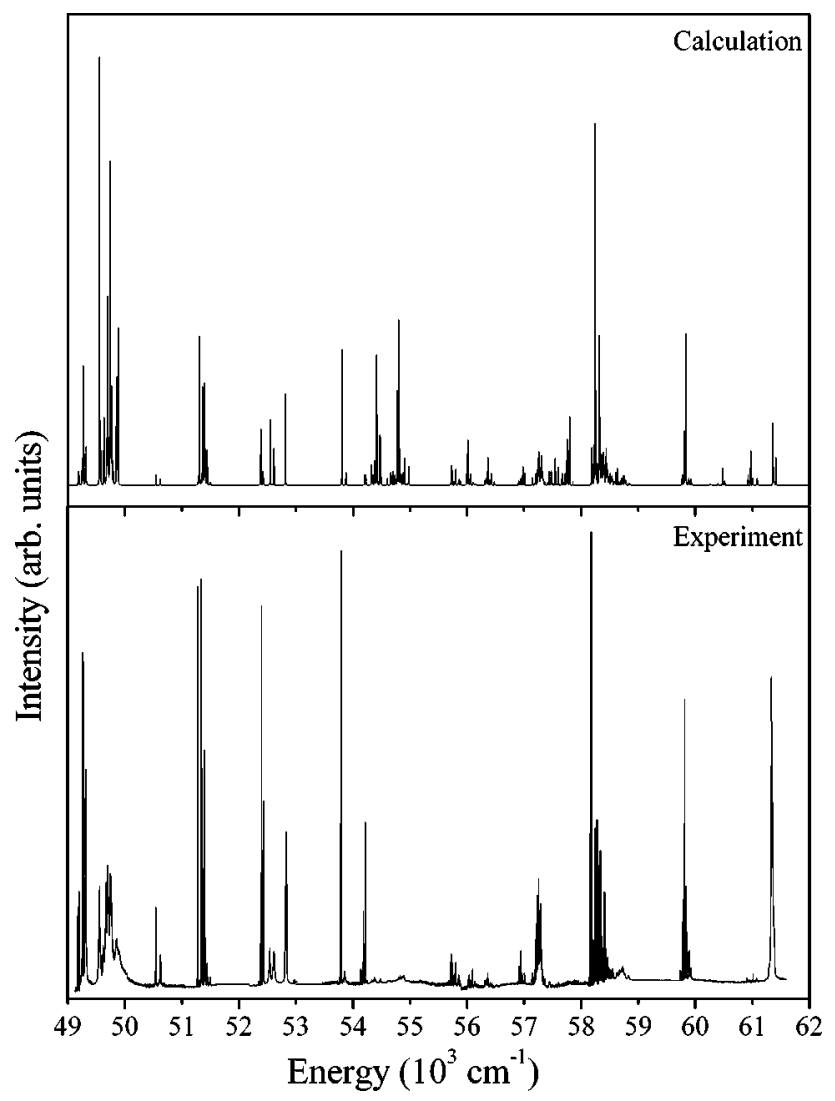

FIG. 7. Calculated and experimentally observed excited state excitation spectra of $\mathrm{LaF}_{3}: \mathrm{Gd}^{3+}$ monitoring ${ }^{6} I_{7 / 2}$ emission at $10 \mathrm{~K}$ for the complete (corrected) dye range of $49000-62000 \mathrm{~cm}^{-1}$. 


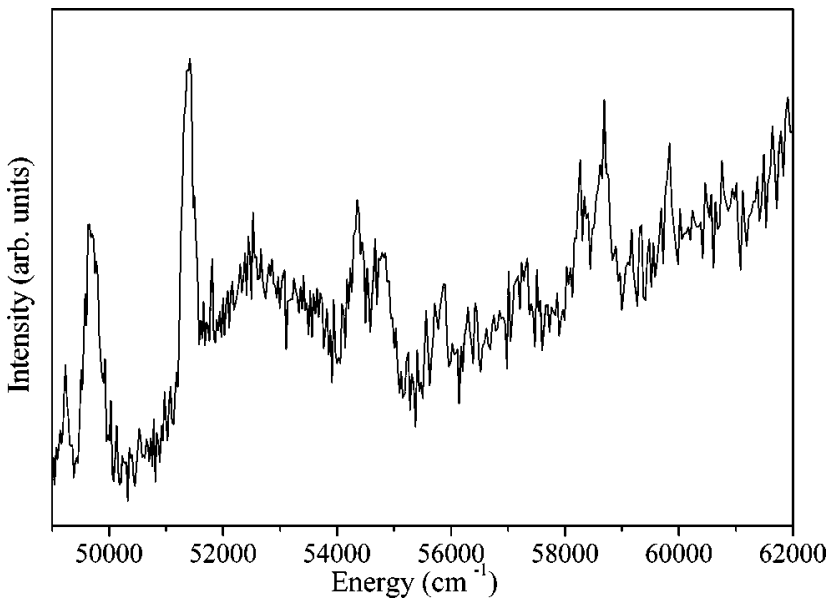

FIG. 8. Excitation spectrum of $\mathrm{LaF}_{3}: \mathrm{Gd}^{3+} 0.5 \%$ crystal recorded at the DESY synchrotron monitoring ${ }^{6} P_{7 / 2}$ emission at $10 \mathrm{~K}$.

small discrepancies in intensity and causes the small shifts of baseline in the spectrum.

The upper spectrum in Fig. 7 shows the calculated excited state excitation spectrum in the same energy range based on the newly fitted parameter values. Section IV B will discuss the fitting method used. For the simulated spectrum the temperature has been set to $10 \mathrm{~K}$ and a linewidth of $1.5 \mathrm{~cm}^{-1}$ was chosen.

The general agreement between the calculated spectrum and the observed spectrum is good. The strong lines in the calculated spectrum show up as strong lines in the experiment and lines for which a small intensity is calculated are weak or not observed. However, not all experimentally observed absorption intensities show a good agreement with the intensities in the calculated spectrum. Some of the absorption lines are broadened, while in the calculated spectrum the same linewidth is given to every absorption line leading to (peak)-intensities that are higher in the calculation than in the experiment. This can explain why the peak intensity for the broadened lines just below $50000 \mathrm{~cm}^{-1}$ is lower than for the sharp lines in the calculated spectrum for this spectral region. The calculated intensity of the absorption lines around $50500 \mathrm{~cm}^{-1}$ and $57250 \mathrm{~cm}^{-1}$ is remarkably lower than the experimental intensity. Also, the absorption lines around $61400 \mathrm{~cm}^{-1}$ are lower in the calculation when compared to the experimentally observed intensities. These differences may be caused to some extent by a wrong correction file for the output of the dye laser, especially for the UV region, where we used the relative efficiencies of the dyes as a correction factor. The discrepancy between the calculated and observed intensities for some of the lines is too large to be explained by correction errors for the dye laser intensities. Further improvements in the model used may resolve the discrepancy.

For comparison, Fig. 8 shows the one-photon excitation spectrum of the same crystal of $\mathrm{LaF}_{3}: \mathrm{Gd}^{3+} 0.5 \%$ recorded at the DESY synchrotron, monitoring ${ }^{6} P_{7 / 2}$ emission at $311 \mathrm{~nm}$. In this spectrum only groups of energy levels can be observed, the individual splitting by the crystal field cannot be resolved. The poor resolution and low signal to noise ratio in this spectrum is related to the low $\mathrm{Gd}^{3+}$ concentration $(0.5 \%)$ and defect absorption bands in the VUV. In the one-photon excitation spectrum the excitation lines that can be observed do coincide with the stronger excitation lines in the excited state excitation spectrum.

\section{B. Fitting of parameters values}

There is a clear difference between the calculated and observed energies. The energy difference ranges from $\sim 100 \mathrm{~cm}^{-1}$ for the lower energy VUV levels to $180 \mathrm{~cm}^{-1}$ for the highest energy levels. Clearly, the parameter values obtained from the lower energy level fitting need to be improved to explain the positions of the high energy levels. To do this, the experimentally observed high energy levels are used to determine new and better parameter values. The fitting procedure for the higher energy levels is, however, not straightforward.

Although many energy levels were obtained by experiment, not all of them can be used in the parameter fitting procedure. Experimentally obtained energies have to be linked to specific energy levels in the calculation. Since the energy difference between the calculation based on Carnall's parameters and experiment increases when going to higher energies, assignment of a single level that matches in energy with a calculated energy level may not correspond to a correct assignment and could cause the fit to shift into the wrong direction.

Whenever possible complete multiplets have to be assigned. In the region $49000-62000 \mathrm{~cm}^{-1}$ the density of levels is still low enough to have energy gaps of about $1000 \mathrm{~cm}^{-1}$ which facilitates the correct assignment of complete multiplets. Unfortunately, due to the broadening of levels or the occurrence of overlapping absorptions it was not always possible to assign a complete multiplet unambiguously. Overlap of multiplets also occurs in the calculated energy levels, which renders assignment difficult.

In order to extract new, and better, parameters describing the energy level structure for $\mathrm{Gd}^{3+}$ in $\mathrm{LaF}_{3}$ it is important to carefully choose the energy levels that can be assigned unambiguously. Following the reasoning discussed above, 83 levels in the vacuum ultraviolet that were resolved by excited state excitation were used in the fitting procedure.

A second choice concerns the parameters that can vary in the fitting procedure. Allowing all parameters to vary freely often results in values that are not meaningful (e.g., wrong sign). To avoid this, the number of parameters needs to be limited to parameters that have a clear influence on the energy levels studied and fix the ratio of other parameters to established ratios.

First we consider the three-body interaction parameters $T^{i}$. The Crosswhite input files ${ }^{13}$ for the energy level program contain the matrix elements of the three electron operators $t_{i},{ }^{14}$ and other electronic free-ion operators. The diagonal elements were all zero except for the elements of $t_{2}$. If no mixing of states would occur, $T^{2}$ would be the only threebody interaction parameter having effect on the energies. Since mixing of states cannot be neglected, the off-diagonal elements for the three-electron operators should be taken into account. This is more complicated, especially because of the 


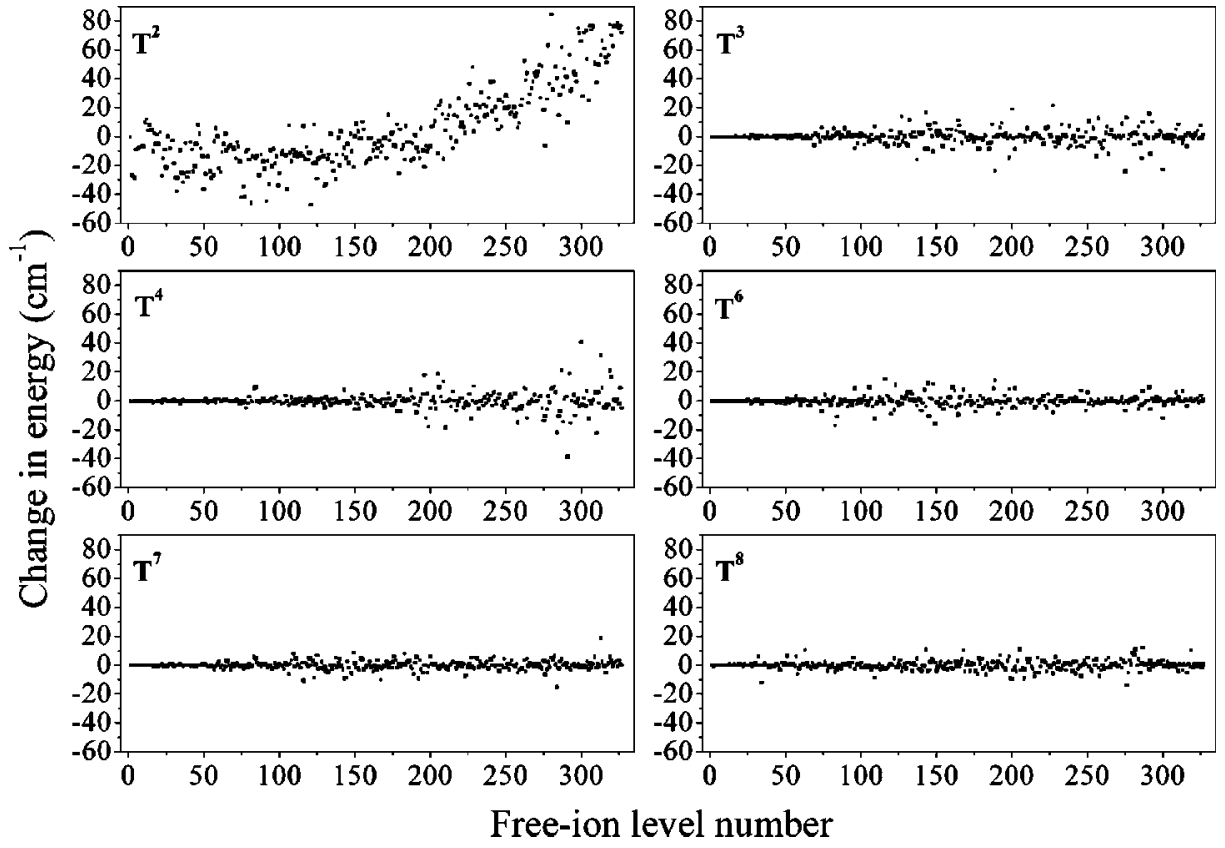

FIG. 9. Changes in energy of the free-ion levels calculated for an increase of $20 \mathrm{~cm}^{-1}$ for the three-body interaction parameters $T^{2}$ to $T^{8}$. All vertical scales are set to the same energy range. large number of levels that have the same $J$ value that can mix with each other. To get an impression of the relative influence of the $T^{n}$ parameters on the energies, the free-ion levels of gadolinium were calculated while the value of each one of the six three-body interaction parameters was increased separately by $20 \mathrm{~cm}^{-1}$. In Fig. 9 the change in energy of the free ion levels is plotted against the free ion energy level number. The vertical scale was set the same for each plot to facilitate comparison.

In our measurements the energy levels up to number 52 [the ${ }^{4} F(4)_{7 / 2}$ level around $61300 \mathrm{~cm}^{-1}$ ] were probed using excited state excitation. For these levels only $T^{2}$ shows a large influence on nearly all the calculated energies, whereas for all other $T^{i}$ parameters the energies of the levels up to level 52 are not affected by the change in the value for $T$ \{except for $T^{8}$ which gives a significant change in energy only for levels $32\left[{ }^{4} \mathrm{H}(2)_{7 / 2}\right]$ and $\left.34\left[{ }^{4} \mathrm{H}(2)_{13 / 2}\right]\right\}$. Based on this, it is reasonable to only allow $T^{2}$ to vary in the energy level fitting while keeping the other $T$ 's at constant values. The only way to fit the values of the $T^{3}$ up to $T^{8}$ for gadolinium in order to obtain more accurate values would be to include even higher energy levels. Starting at about free ion level 100 the $T^{3}, T^{4}$, and $T^{6}$ parameters have enough influence on the energy to fit them to experimental data. However, these levels are situated at $73000 \mathrm{~cm}^{-1}$ and higher and have never been reported for $\mathrm{Gd}^{3+}$ in $\mathrm{LaF}_{3}$. The density of levels is high at this energy, and energy differences between consecutive levels are generally about $10 \mathrm{~cm}^{-1}$. Fast relaxation will broaden these levels in such an extent that assignment of these levels will be impossible. Moreover, at these energies most energy levels are spin quartet and doublet states. Therefore it does not appear to be possible to improve the values of $T^{3}$ to $T^{8}$ for gadolinium.

The higher order magnetic interaction parameters $M$ and $P$ were allowed to vary. Following standard convention, ${ }^{2}$ the $M^{2}$ and $M^{4}$ were kept at fixed ratios of 0.56 and 0.31 to $M^{0}$, the same was done with $P^{4}$ and $P^{6}$ with respect to $P^{2}$ with ratios of 0.5 and 0.1 , respectively. ${ }^{2}$
For the crystal field parameters $B_{q}^{k}$ (in Wybourne normalization $)^{15}$ the values of $k$ are restricted to 2,4 , and 6 for $4 f$-electron configurations. For $C_{2 v}$, which is the effective site symmetry used for $\mathrm{LaF}_{3}$, the restrictions for $q$ are $q$ =even and $0 \leqslant q \leqslant k$. In our calculations the $C_{2}$ site symmetry is approximated by $C_{2 v}$ by constraining all of the crystal field parameters to be real. This approximation has an extremely small effect on the energy levels and is almost universally adopted ${ }^{2}$ in fitting spectra in $\mathrm{LaF}_{3}$. In fact, it is very difficult to obtain consistent values of crystal field parameters if the full $C_{2}$ symmetry is considered. ${ }^{16}$ Besides this one-electron crystal field interaction we also included twoelectron correlation crystal field parameters $D_{q}^{k}$. It is known for certain lanthanide ions like $\operatorname{Pr}^{3+}$ that the one-electron crystal field parametrization gives an accurate description of the crystal field levels for most multiplets, but not for all of them. ${ }^{17}$ The inclusion of two-electron correlated crystal field interaction was shown to improve the calculated splitting of the anomalously behaving multiplets. ${ }^{18}$ We included the delta-function correlated crystal field parameters $D_{q}^{k}$ for $k$ $=2,4$, and 6 using the delta functions as reported by Lo and Reid. $^{19}$

In addition, for the preliminary calculation, not all parameters were allowed to vary at the same time. When starting with initial parameter values that are far from equilibrium, not putting any restraints on the parameter values may result in parameter values that have the wrong sign or order of magnitude and therefore have lost physical meaning. Initially, only the free-ion parameters were allowed to vary and the crystal field parameters were set to the values listed in Table II which were obtained by fitting to the energy levels up to $50000 \mathrm{~cm}^{-1}$ reported by Carnall et al. ${ }^{2}$ The new set of free-ion parameters thus obtained was used as a basis for a second fitting procedure in which the crystal field parameters were varied. Finally, a fit in which all parameters were allowed to vary freely (except $T^{3}$ to $T^{8}$ which were fixed and $M^{2}, M^{4}, P^{4}$, and $P^{6}$ set to the ratios mentioned above) was performed. 
TABLE VII. Free-ion and crystal-field parameters for $\mathrm{LaF}_{3}: \mathrm{Gd}^{3+}$. Parameters for which no uncertainty is given were not varied. The number of data $N$, standard deviation and root mean square deviation are given at the bottom of the table.

\begin{tabular}{|c|c|c|c|}
\hline Parameter & $\begin{array}{l}\text { All data } \\
\text { value } \\
\left(\mathrm{cm}^{-1}\right)\end{array}$ & $\begin{array}{c}\text { Carnall's data } \\
\text { value } \\
\left(\mathrm{cm}^{-1}\right)\end{array}$ & $\begin{array}{c}\text { Present data } \\
\text { value } \\
\left(\mathrm{cm}^{-1}\right)\end{array}$ \\
\hline$E_{\mathrm{AVG}}$ & $88025(7)$ & $87812(10)$ & $87989(5)$ \\
\hline$F^{2}$ & $86065(67)$ & $85592(18)$ & $85798(82)$ \\
\hline$F^{4}$ & 61499(95) & 61015(29) & $61529(103)$ \\
\hline$F_{6}$ & $44832(116)$ & $44745(25)$ & 44633(137) \\
\hline$\alpha$ & $19.33(0.17)$ & $19.13(0.09)$ & $18.97(0.19)$ \\
\hline$\beta$ & $-528(26)$ & -600 & $-520(23)$ \\
\hline$\gamma$ & $1450(24)$ & 1575 & $1490(23)$ \\
\hline$T^{2}$ & 263(19) & 300 & 288 \\
\hline$T^{3}$ & 42 & 42 & 42 \\
\hline$T^{4}$ & 62 & 62 & 62 \\
\hline$T^{6}$ & -295 & -295 & -295 \\
\hline$T^{7}$ & 350 & 350 & 350 \\
\hline$T^{8}$ & 310 & 310 & 310 \\
\hline$\zeta$ & $1516(2)$ & $1499(2)$ & $1517(2)$ \\
\hline$M^{0}$ & $2.85(0.12)$ & $3.20(0.03)$ & $2.75(0.29)$ \\
\hline$P^{2}$ & $538(37)$ & 676 & $520(51)$ \\
\hline$B_{0}^{2}$ & $-223(36)$ & $-212(14)$ & $-198(32)$ \\
\hline$B_{0}^{4}$ & $622(171)$ & $579(40)$ & $909(96)$ \\
\hline$B_{0}^{6}$ & $441(143)$ & $292(22)$ & 143(89) \\
\hline$B_{2}^{2}$ & $-92(27)$ & $0.4286 B_{0}^{2}$ & $-117(19)$ \\
\hline$B_{2}^{4}$ & 217(93) & $0.5629 B_{0}^{4}$ & 193(82) \\
\hline$B_{4}^{4}$ & $556(95)$ & $0.7483 B_{0}^{4}$ & $343(88)$ \\
\hline$B_{2}^{6}$ & $-720(89)$ & $-2.575 B_{0}^{6}$ & $-891(60)$ \\
\hline$B_{4}^{6}$ & $-331(86)$ & $-0.7286 B_{0}^{6}$ & $-153(89)$ \\
\hline$B_{6}^{6}$ & $-598(103)$ & $-1.8179 B_{0}^{6}$ & $-520(87)$ \\
\hline$D_{0}^{2}$ & $1.02(0.88)$ & & $1.29(1.57)$ \\
\hline$D_{0}^{4}$ & $-3.01(0.93)$ & & $-2.53(0.98)$ \\
\hline$D_{0}^{6}$ & $-0.46(0.55)$ & & $-0.22(0.45)$ \\
\hline$N$ & 133 & 70 & 83 \\
\hline$\sigma$ & 16.1 & 8.0 & 11.2 \\
\hline $\mathrm{rms}$ & 14.6 & 7.4 & 10.1 \\
\hline
\end{tabular}

On the basis of the newly observed energy levels in the VUV two fittings were done following the strategy discussed above, in addition to the fit based on the UV energy levels reported by Carnall et al. The resulting sets of parameters and their uncertainties are collected in Table VII. In the first column the parameters are given based on fitting all 133 experimentally observed levels. The levels included are the levels up to $49000 \mathrm{~cm}^{-1}$ as reported by Carnall et al. ${ }^{2}$ and the presently reported VUV energy levels. The quality of the fit is reflected by the standard deviation $\sigma\left(16 \mathrm{~cm}^{-1}\right)$ and root mean square deviation $\left(14.6 \mathrm{~cm}^{-1}\right)$. The second column gives the parameters obtained for the best fit for the lower energy levels (up to $49000 \mathrm{~cm}^{-1}$ ) while the third column contains the values for the parameters obtained by fitting the high energy (VUV) levels.
The quality of the fits for either the higher energy levels or the lower energy levels is very good which is demonstrated by the small root mean square deviation and standard deviation for these fits (around $10 \mathrm{~cm}^{-1}$ ). For the fit including all energy levels the agreement is not as good which is not only clear from the higher numbers for $\sigma$ and rms but also from the fact that the ground state is calculated at $-108 \mathrm{~cm}^{-1}$ with the parameters in the first column in Table VII. The origin for the discrepancy between the parameters obtained from fitting the experimentally observed energy levels in the two spectral regions is not clear. Calibration errors of the experimental setups can be excluded. With the dye laser used here for measuring the VUV energy levels of $\mathrm{Gd}^{3+}$ by excited state excitation we also measured the UV levels for $\mathrm{Gd}^{3+}$ in $\mathrm{LaF}_{3}$ using one-photon and two-photon excitation. Our experiments give energies for the UV levels that agree within approximately $1 \mathrm{~cm}^{-1}$ with the values found by Carnall et al. ${ }^{2}$ and also with the energies reported by Downer obtained by two-photon experiments on $\mathrm{LaF}_{3}: \mathrm{Gd}^{3+3}$.

At this point it is only possible to speculate about the origin of the discrepancy. Possibly the strong intermixing of states in the VUV region, where there is an overlap between many closely spaced multiplets, is not well reproduced by the presently used model and further refinement of the model

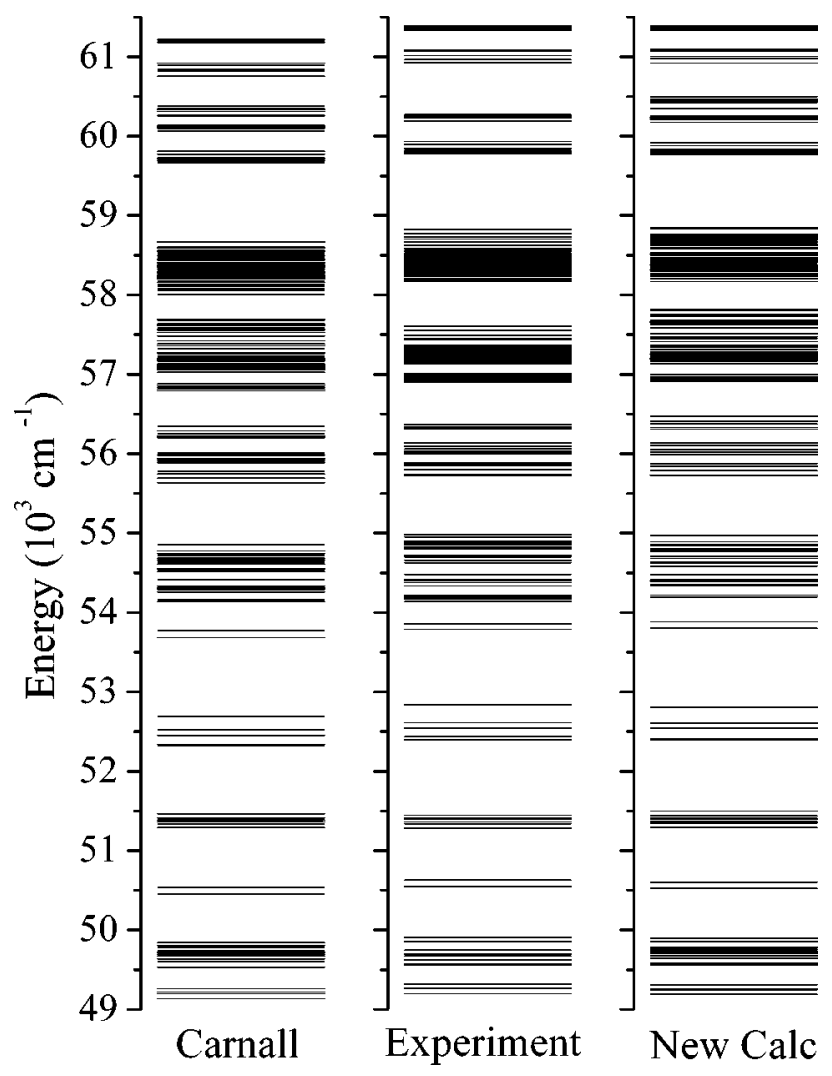

FIG. 10. Calculated and experimentally observed energy levels for $\mathrm{Gd}^{3+}$ in $\mathrm{LaF}_{3}$ using excited state excitation. The left column shows the energies calculated using the parameters by Carnall et al. (Ref. 2), the middle column displays all experimentally observed energy levels and the right column shows the energy level diagram with the new fit parameters. 
is required. This may also explain some of the differences observed between the calculated and experimentally observed intensities for transitions in the VUV (see Fig. 7).

The agreement between the calculated VUV energy levels and the experimentally observed energies is good when the parameter values from the new fit are used. In Fig. 10 this is illustrated. In this figure the calculated energy level scheme for $\mathrm{Gd}^{3+}$ in $\mathrm{LaF}_{3}$ is shown for both sets of parameters together with all experimentally observed energy levels, including the energy levels that have not been used in the fitting of the parameters. The agreement between the experimentally observed levels and the energy level structure calculated with the new set of fitting parameters (given in the third column of Table VII) is very good while the energy level structure based on calculations with the parameters from fitting of the data reported by Carnall et al. (given in the second column of Table VII) cannot explain the observed energy level structure. Note that some of the calculated energy levels in the energy level diagram based on the new parameters are not experimentally observed. The reason for the absence in the experimental spectra is that the excited state excitation transitions to these levels is forbidden due to selection rules. For example, since transitions to levels with $J$-values higher than 19/2 are formally forbidden the energy levels that are calculated in the energy region around 57700 and $60400 \mathrm{~cm}^{-1}$ are not observed in the experiment.

\section{CONCLUSIONS}

Excited state excitation is shown to be a viable method to measure the energy levels of lanthanide ions in the vacuum ultraviolet region with a high accuracy. For $\mathrm{Gd}^{3+}$ in $\mathrm{LaF}_{3}$ many VUV levels up to $62000 \mathrm{~cm}^{-1}$ are reported with a resolution of $\sim 1 \mathrm{~cm}^{-1}$. The energy levels measured were used as a basis for the determination of more accurate parameter values for the energy level structure of $\mathrm{Gd}_{3+}$ in $\mathrm{LaF}_{3}$. It was not possible to obtain a parameter set that gives a good agreement for both the lower energy UV levels (below $50000 \mathrm{~cm}^{-1}$ ) and the presently obtained VUV levels. Intensity calculations reproduce the observed line strengths for most absorption lines, but for some absorptions there are differences between calculated and observed intensities. The inability of the calculations to reproduce the energy level structure and line intensities for the full spectral region in good agreement with experiment may be related to the strong overlap and intermixing of states in areas where the energy levels are closely spaced. Further improvements in the energy level calculation model may resolve this issue.
*Electronic address: p.s.peijzel@chem.uu.nl

${ }^{1}$ G. H. Dieke, Spectra and Energy Levels of Rare Earth Ions in Crystals (Interscience, New York, 1968).

${ }^{2}$ W. T. Carnall, G. L. Goodman, K. Rajnak, and R. S. Rana, A Systematic Analysis of the Spectra of the Lanthanides Doped into Single Crystal $\mathrm{LaF}_{3}$ (Argonne National Laboratory, Argonne, IL, 1988).

${ }^{3}$ M. Dagenais, M. Downer, R. Neumann, and N. Bloembergen, Phys. Rev. Lett. 46, 561 (1981).

${ }^{4}$ M. C. Downer and A. Bivas, Phys. Rev. B 28, 3677 (1983).

${ }^{5}$ P. A. Tanner, V. V. Ravi Kanth Kumar, C. K. Jayasankar, and M. F. Reid, J. Alloys Compd. 215, 349 (1994).

${ }^{6}$ K. A. Schoene, J. R. Quagliano, and F. S. Richardson, Inorg. Chem. 30, 3803 (1991).

${ }^{7}$ J. Hölsä, R. J. Lamminmäki, M. Lastusaari, and P. Porcher, J. Alloys Compd. 323-324, 811 (2001).

${ }^{8}$ R. T. Wegh, H. Donker, A. Meijerink, R. J. Lamminmäki, and J. Hölsä, Phys. Rev. B 56, 13841 (1997).

${ }^{9}$ R. T. Wegh, A. Meijerink, R. J. Lamminmäki, and J. Hölsä, J.
Lumin. 87-89, 1002 (2000).

${ }^{10}$ R. T. Wegh, E. V. D. van Loef, G. W. Burdick, and A. Meijerink, Mol. Phys. 101, 1047 (2003).

${ }^{11}$ P. S. Peijzel, R. T. Wegh, A. Meijerink, J. Hölsä, and R. J. Lamminmäki, Opt. Commun. 204, 195 (2002).

${ }^{12}$ P. S. Peijzel, A. Meijerink, R. T. Wegh, M. F. Reid, and G. W. Burdick, J. Solid State Chem. (to be published).

${ }^{13}$ H. M. Crosswhite and H. Crosswhite, J. Opt. Soc. Am. B 1, 246 (1984).

${ }^{14}$ H. Crosswhite, H. M. Crosswhite, and B. R. Judd, Phys. Rev. 174, 89 (1968).

${ }^{15}$ B. G. Wybourne, Spectroscopic Properties of Rare Earths (Interscience, New York, 1965).

${ }^{16}$ Y. Y. Yeung and M. F. Reid, J. Less-Common Met. 148, 213 (1989).

${ }^{17}$ Y. Y. Yeung and D. J. Newman, J. Chem. Phys. 86, 6717 (1987).

${ }^{18}$ G. W. Burdick and F. S. Richardson, Chem. Phys. 228, 81 (1998).

${ }^{19}$ T. S. Lo and M. F. Reid, J. Alloys Compd. 193, 180 (1993). 